\title{
Seek and you shall find...
}

Essential thrombocythemia and primary myelofibrosis are common types of myeloproliferative neoplasms (MPNs). Mutational activation of Janus kinase 2 (JAK2) is associated with $50-60 \%$ of cases of these MPNs; the underlying cause of approximately another $30 \%$ of these MPN cases is unknown. Two papers now report that mutations in calreticulin (CALR) occur in the majority of patients with these MPNs in which JAK2 is not activated (JAK2- disease).

Klampfl et al. carried out wholeexome sequencing of matched samples from six patients with primary myelofibrosis and found CALR mutations in all tumour samples. Two of the samples had deletions in exon 9 (a 52 bp deletion was classified as the type 1 mutation) and four of the samples had a $5 \mathrm{bp}$ insertion in exon 9 (the type 2 mutation). Furthermore, patients with CALR mutations, including those from an additional cohort, were $J A K 2^{-}$and CALR mutation occurred in $88 \%$ of these JAK2- MPNs.

In total, Klampfl et al. found 36 different CALR mutations (mostly type 1 and type 2 mutations), all of which caused a frameshift to alternative reading frame 1 , which was predicted to alter the carboxy-terminal domain. Type 1 mutations were significantly the underlying cause of approximately another 30\% of these MPN cases is unknown. associated with primary myelofibrosis, indicating that the two types of mutation might have different effects. Exogenous expression of a type 1-mutant protein conferred interleukin-3 (IL-3)independent growth to cells, which seemed to be caused by the activation of JAK-signal transducer and activator of transcription 5 (STAT5) signalling. In total, the authors studied 1,215 patients with essential thrombocythemia or primary myelofibrosis; $23.5 \%$ of these patients had CALR mutations, and patients with $C A L R^{+}$ disease had a longer overall survival compared with those with $J A K 2^{+}$disease.

In the other paper, Nangalia et al. carried out whole-exome sequencing of $151 \mathrm{MPN}$ samples and found CALR exon 9 insertions and deletions in 26 of 31 patients with $J A K 2^{-}$essential thrombocythemia or primary myelofibrosis; similar results were found in a larger cohort. The type 1 and type 2 mutations were the most common, and the type 1 mutation was again most prevalent in the primary myelofibrosis samples. Interestingly, these authors found that $C A L R^{+}$ essential thrombocythemia had a higher incidence of transformation to myelofibrosis than the $J A K 2^{+}$disease. Further analyses also indicated that CALR mutation occurred in the haematopoietic stem cell compartment and that this was an early event in the five patients who were tested, which indicates that it might be a driver mutation.

Nangalia et al. found 19 different CALR mutations and they also predicted that each mutation affected the carboxy-terminal domain. These mutations were predicted to cause the loss of a KDEL motif, which can determine localization in the endoplasmic reticulum, and to variably affect the acidity of this domain. However, exogenous expression of the type 1 and type 2 CALR mutants in HEK 293 T cells

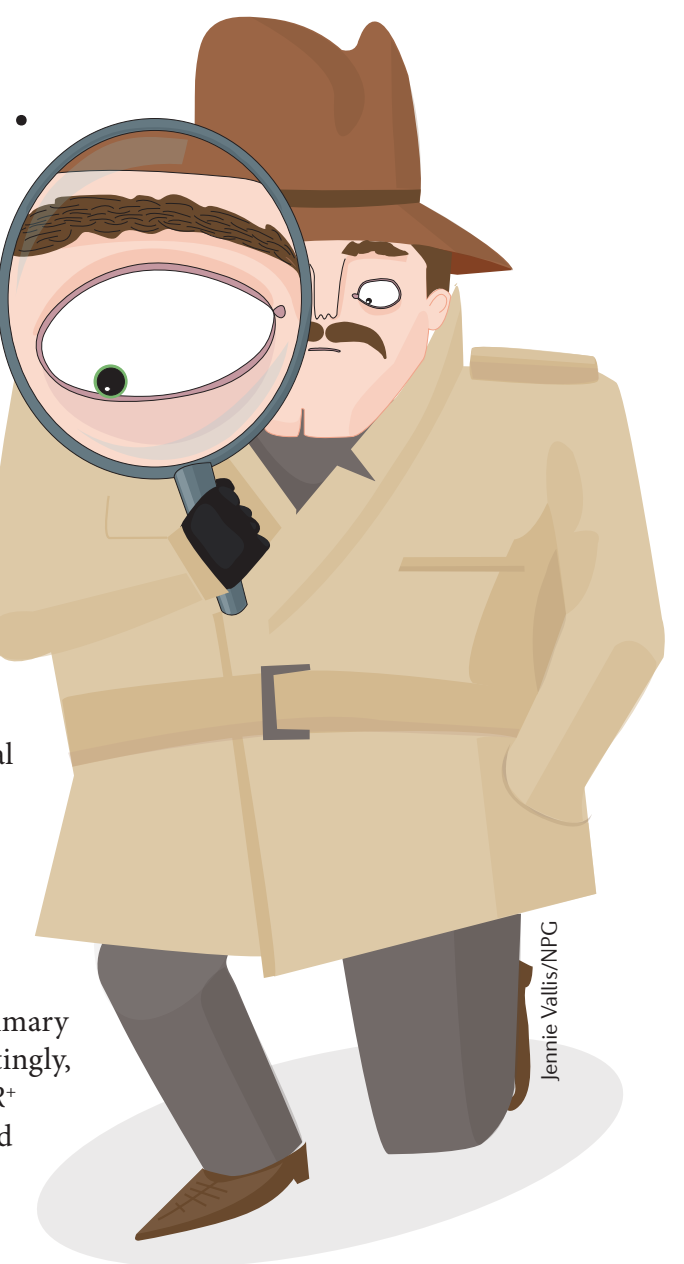

revealed that the subcellular localization of CALR was not affected by mutation; thus, further work is needed to understand why type 1 and type 2 mutations of CALR are oncogenic.

Together, these papers add to the list of underlying causal alterations of MPNs. It will be interesting to discover whether these alterations have any bearing on therapy.

Gemma K. Alderton

ORIGINAL RESEARCH PAPERS Klampfl, T. et al.

Somatic mutations of calreticulin in myeloproliferative neoplasms. N. Engl. J. Med. 369, 2379-2390 (2013) | Nangalia, J. et al. Somatic CALR mutations in myeloproliferative neoplasms with nonmutated JAK2. N. Engl. J. Med. 369, 2391-2405 (2013) 